\title{
Robot Assistant for Dental Implantology
}

\author{
R. Boesecke ${ }^{1}$, J. Brief ${ }^{2}$, J. Raczkowsky $^{4}$, O. Schorr ${ }^{4}$, S. Daueber ${ }^{3}$, \\ R. Krempien ${ }^{4}$, M. Treiber ${ }^{4}$, T. Wetter ${ }^{5}$ and S. Hassfeld ${ }^{2}$ \\ ${ }^{1}$ TaMed GmbH, Hufschmiedstr. 1/4, D-69168 Wiesloch, Germany \\ r.boesecke@praezis.com \\ ${ }^{2}$ Department of Oral and Maxillofacial Surgery, University Hospital Heidelberg \\ ${ }^{3}$ Department of Radiology, University Hospital Heidelberg \\ ${ }^{4}$ Institute of Real-Time Computer Systems \& Robotics, University of Karlsruhe \\ ${ }^{5}$ Department of Medical Informatics, University Hospital Heidelberg
}

\begin{abstract}
We introduce a method to apply a preoperative 3D plan for inserting dental implants with an assisting medical robot. The treatment plan is based on the $3 \mathrm{D}$ visualization of the CT data of the patient's maxilla and mandible, and supplies the location of the implants in the patient's coordinates. The plan is then transferred to the surgical robot's coordinate system. The robot guides the tool, a drill guide. Position, orientation, and depth of the initial drilling is defined with the tool held by the robot while the surgeon drills. The robot assists the dentist, and the optimal treatment plan will be applied directly to the patient.
\end{abstract}

\section{Introduction}

The quality of the insertion of dental implants as well as possible risks depend not only on the surgery planning, but as well on performing the surgery as exact as possible according to the treatment plan.

Methods of Computer Aided Surgery are used to plan for these parameters. To apply the so obtained treatment plan, we introduced an assisting robot system at the University Hospital of Heidelberg as a prototype system. We evaluated the system accuracy performing 16 operation plans with phantom mandibles.

\section{Material and Method}

A robot system from Medical Intelligence, Schwabmünchen, Germany, with a reach of $700 \mathrm{~mm}$ was used. The PC based software TomoRob from TaMed, Wiesloch, Germany, allows for simulation, visualization, and control of the robot. The position and orientation of the implants was planned based on 3D CT data with TomoRob. Visualization of the robot, the patient couch, the patient, tools and implants are used for planning and control of the surgery. The trajectory planning is based on linear interpolation of start and end point and points between. A collision detection algorithm is integrated to warn during the computer simulation of the surgery. 

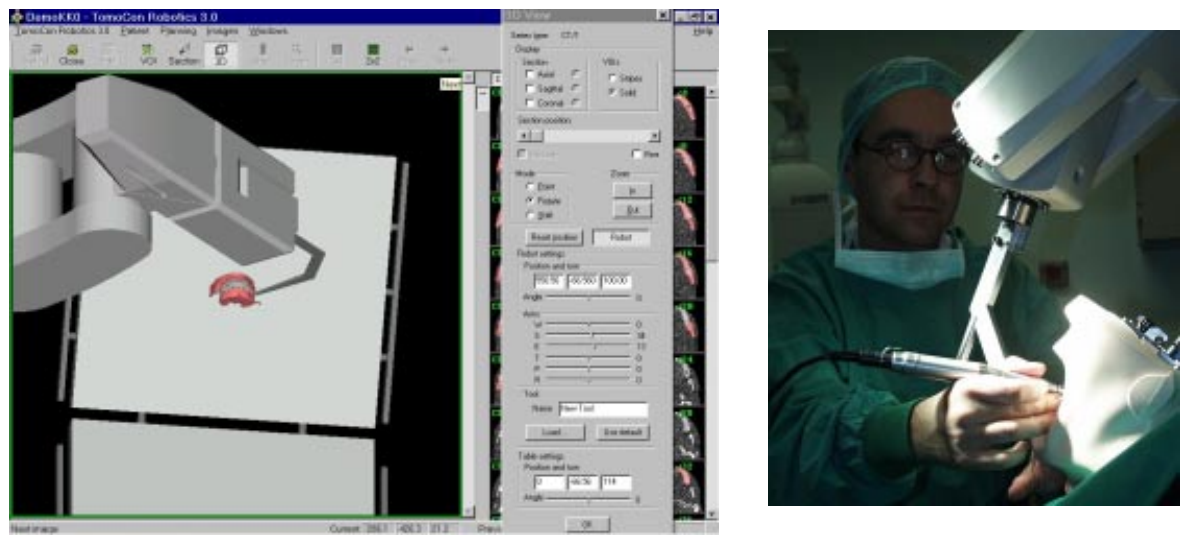

Fig. 1. Simulation before surgery (left). The robot is in surgery position holding the drill guide (right).

After acceptance of the plan by the surgeon, the robot starts to move under control of TomoRob. The communication with the robot controller is based on a standard communication protocol.

With pointing with the robot's pointer to a minimum of three artificial landmarks, the robots coordinate systems, the image coordinate system and the patients coordinate system were referenced. We used the corners of a LEGO block, also visible in the CT data, as landmarks. The fixation of the patients jaws during treatment is still necessary at this stage.

In the current configuration, the robot is mounted on a trolly, which holds the robot, the small robot controller and the laptop computer for TomoRob. The trolly can be connected to a standard operation table with rigid mountings.

Initial drilling was performed by the surgeon. The robot assisted by holding a drill template according to the preoperative determined position data of the implants.

For the 16 performed model operations with altogether 48 drills for implants, post OP CT examinations were performed without the implants in place to avoid artifacts. Then, implant top (bone entrance) positions and implant end positions (implant apex) were compared against the original plan.

\section{Results}

First and preliminary results show that, after performing the fusion of pre and post OP CT, deviations of about 1 to $2 \mathrm{~mm}$ where found for the implant top and implant apex positions. The time saving effect expected from using robots in surgery depends on the effectiveness of the referencing procedure and the optimization and streamlining of the software for practical use. 